\title{
A todas luces, una experiencia única entre ciencia y arte
}

\author{
Isaías Hernández Valencia
}

\section{Resumen}

Este artículo tiene como objetivo presentar la experiencia que se ha vivido durante el desarrollo y puesta en marcha de las actividades del proyecto $A$ todas luces, diálogo de saberes entre ciencia y arte; y exponer las bondades, diferencias y retos que se tuvieron al recorrer seis escuelas de nivel primaria, de primer grado a sexto grado, en diversos estados de la república mexicana. Se expondrán, también, algunas reflexiones y comparaciones con otras actividades que el Museo de la luz hace regularmente fuera de sus muros en otros contextos con propósitos específicos; para que, de esa manera, se vea la diferencia y potencialidad de haber trabajado divulgadores de la ciencia y artistas plásticos en un proyecto en común.

Palabras clave: ciencia, arte, educación.

\section{A todAS LUCES, A UNIQUE EXPERIENCE BETWEEN SCIENCE AND ART}

\begin{abstract}
This article aims to present the experience that has been lived during the development and implementation of the activities of the project "Clearly, dialogue of knowledge between science and art"; and to expose the kindness, differences and challenges that were had when crossing six primary schools, from first grade to sixth grade, in different states of the Mexican Republic. Some reflections and comparisons with other activities that the museum regularly makes outside its walls in other contexts with specific purposes will be exposed; so that, in this way, we can see the difference and potentiality of having worked for science disseminators and plastic artists in a common project.
\end{abstract}

Key words: science, art, education. 
Físico y divulgador de la ciencia. Es jefe de Servicios Académicos del Museo de la Luz, perteneciente a la Dirección General de Divulgación de la Ciencia de la Universidad Nacional Autónoma de México (UNAM).

\section{Una primera reflexión entre ciencia y arte}

Antes de comenzar a describir la experiencia vivida en el proyecto, quisiera aportar algunas reflexiones e ideas generales que se tienen de las disciplinas científicas y artísticas.

El arte y la ciencia son necesariamente diferentes desde el punto de vista del estudio y la difusión del saber. La ciencia intenta comprender el mundo a partir de un conocimiento acumulado en el tiempo, basándose en resultados reproducibles y en hechos demostrables y contrastados; se esfuerza en buscar la objetividad. Las humanidades, disciplinas académicas que observan la condición humana, utilizan métodos principalmente analíticos, críticos y especulativos. Las artes escénicas, visuales y literarias expresan el mundo a partir de las experiencias idiosincrásicas, la intuición, el movimiento y las metáforas. Los artistas suelen inspirarse en el pasado, pero la "verdad" de una obra de arte no se halla a través de una investigación sistemática que conduzca a hechos acumulativos y reproducibles; se trata, a menudo, de algo subjetivo y sensorial.

Tabla 1.

Diferencias entre arte $y$ ciencia

\begin{tabular}{|c|c|c|}
\hline \multirow{6}{*}{ Arte } & • & Busca respuestas estéticas. \\
\hline & $\cdot$ & Está basado en la emoción y la intuición. \\
\hline & . & Es idiosincrático. \\
\hline & - & Se alimenta de la comunicación visual o sonora. \\
\hline & • & Es evocativo. \\
\hline & & Se basa en valores que rompen tradiciones. \\
\hline \multirow{6}{*}{ Ciencia } & • & Busca el conocimiento y la comprensión de todo. \\
\hline & · & Se basa en el uso de la razón. \\
\hline & . & Vive de la normativa. \\
\hline & • & Requiere una narrativa para su comprensión. \\
\hline & & Su estrategia es explicativa. \\
\hline & & $\begin{array}{l}\text { Se alimenta de valores sistemáticos basados en la tradición y en el } \\
\text { cumplimiento de las normas. }\end{array}$ \\
\hline
\end{tabular}


Sin embargo, ambas disciplinas pueden llegar a estar profundamente conectadas ya que, a un nivel básico, todos nosotros exploramos fenómenos y experiencias vividas para intentar comprender la realidad y las potencialidades que pueden existir bajo ellas.

Aún más evidentes son las similitudes fundamentales compartidas por artistas y científicos. Tanto unos como otros se describen, en general, como creativos, intuitivos, disciplinados, lógicos e inspirados en sus actividades para comprender el mundo. Valoran la observación cuidadosa de sus entornos para recopilar información a través de los sentidos.

- Aprecian la creatividad.

- Proponen introducir cambios, innovaciones o mejoras sobre lo que existe.

- Utilizan modelos abstractos para entender el mundo.

- Aspiran a crear obras de relevancia universal.

Además, analizando las habilidades y estilos de vida de las personas exitosas en las artes, humanidades, ciencias y tecnología para entender qué es lo que hace que piensen creativamente, innoven y triunfen dentro, e incluso fuera, de sus disciplinas, encontramos que esas personas exhiben o exhibían el mismo conjunto de habilidades imaginativas, basta pensar en artistas como Picasso o físicos como Richard Feynman, sólo por mencionar un ejemplo.

\section{8}

\section{Como el salón estaba} oscurecido, apagábamos todas las luces y veían que lo que habían hecho en el taller requería de luz para verlo, de hecho, se requiere de luz para poder ver todo lo que nos rodea.

Por otro lado, las actividades planeadas y la secuencia didáctica tienen como propósito que, en el proceso, la ciencia informe sobre el arte y ésta sobre la ciencia.

\section{Descripción de las actividades}

En el proyecto A todas luces, diálogo de saberes entre ciencia y arte se busca establecer un puente entre científicos y artistas para que guíen las inquietudes, dudas y propuestas de los niñas, niños y adolescentes, a partir de la observación, la experimentación y la reflexión de lo aprendido sobre el fenómeno de la luz y su aplicación en la creación de un objeto artístico que promueva y transmita el trabajo en equipo, la importancia de la luz para la vida, el cuidado de la naturaleza y su entorno local, el aprovechamiento del conocimiento adquirido para el desarrollo integral del ser humano en su comunidad y el desarrollo sostenible de las futuras generaciones. 
Tabla 2.

Desglose de escuelas, estados, fechas y alumnos que se atendieron en el proyecto
Los objetivos definidos giran en torno a: 1) promover en niñas y niños el interés por conocer de dónde surge la energía solar y que encuentren un uso a ese conocimiento de conocer las explicaciones científicas de fenómenos relacionados con la luz y sus efectos sobre la vida en el planeta, 2) asociar los beneficios y aplicaciones útiles de la radiación solar a la sociedad humana, a través de actividades lúdicas, manipulación de instrumentos y experimentos sencillos, 3) Posibilitar la expresión artística de las niñas y niños desde su perspectiva social y cultural sobre el tema de la luz, 4) vincular el aspecto científico sobre la luz con el aspecto lúdico-creativo en la construcción de objetos con un sentido estético-artístico al realizar un mural o escultura colectiva, y 5) coadyuvar en el crecimiento del acervo cultural y artístico de las escuelas, al donar el objeto artístico elaborado por los estudiantes bajo la guía del artista.

El proyecto estuvo dirigido a estudiantes de educación primaria, cuyas escuelas se encuentran integradas en la Red del Plan de Escuelas Asociadas de la unEsCo. Se escogieron seis escuelas de diferentes estados de la república mexicana (ver tabla 2).

\begin{tabular}{|c|c|c|c|c|}
\hline Escuela primaria & Ciudad & Fechas & Grupos & Alumnos \\
\hline C.E.I Roberto Cabral Del Hoyo. & Zacatecas, Zac. & Mayo 16 al 26 & 18 & 660 \\
\hline \multicolumn{2}{|l|}{$\begin{array}{l}\text { Escuela Práctica Anexa a la Nor- } \\
\text { mal Veracruzana. }\end{array}$} & Junio 2 al 23 & 18 & 495 \\
\hline $\begin{array}{l}\text { Escuela Primaria Francisco J. San- } \\
\text { tamaría. }\end{array}$ & Villahermosa, Tab. & $\begin{array}{l}\text { Agosto } 28 \text { al } 8 \\
\text { de septiembre }\end{array}$ & 12 & 298 \\
\hline $\begin{array}{l}\text { Escuela Urbana \#96 Manuel M. } \\
\text { Diéguez }\end{array}$ & Guadalajara, Jal. & $\begin{array}{l}\text { Septiembre } 18 \\
\text { al } 29\end{array}$ & 12 & 407 \\
\hline $\begin{array}{l}\text { Escuela Primaria Profesora Ob- } \\
\text { dulia Zamora Martínez }\end{array}$ & Monterrey, N.L. & $\begin{array}{l}\text { Octubre } 02 \\
\text { al } 13\end{array}$ & 6 & 149 \\
\hline Escuela Primaria Niños Héroes & Campeche, Camp. & $\begin{array}{l}\text { Noviembre } 13 \\
\text { al } 24\end{array}$ & 13 & 436 \\
\hline & & & 79 & 2445 \\
\hline
\end{tabular}

\section{La manera en que se llevó a cabo}

Para cumplir con los objetivos del proyecto, se planeó que en dos semanas se llevarían a cabo las actividades de ciencia y de arte, para posteriormente terminar con un mural o instalación artística. Divulgadores del Museo de la Luz, en el cual me incluyo, realizamos en la primera semana las actividades de observación, experimentación y talleres con temas de la luz. Los artistas, que forman parte de Asociación de Artistas Plásticos de México A.C. (ARTAC), aunque llegaban con nosotros desde la primera semana, comenzaban a mediados de ésta y terminaban con la presentación de la obra el viernes de la segunda semana. 
Los objetivos se plantearon con la intención de que cuando las niñas y los niños observaran el producto artístico terminado tuvieran una lectura diferente de él al observarla, que no nada más vieran los trazos, las figuras, las formas, los colores, sino que observaran más allá, que con la experiencia que habían vivido supieran de la importancia de la luz en la percepción de los colores, de los mecanismos y fenómenos físicos que están sucediendo en la interacción entre la luz natural o artificial con la obra; que supieran por qué brillan ciertas pinturas cuando se iluminan con un tipo u otro de luz, que conozcan porqué el color que percibimos dependerá del color de la luz incidente, que vean más allá de lo evidente, que vean con conocimiento y con emoción.

Los talleres que se escogieron fueron el Disco de Newton y el Anamorfismo. El hecho de haber escogido estos talleres se debe a que en ambos se utilizan pinturas de diferentes colores, y esto nos da la oportunidad de "jugar" (entendamos jugar como una forma de experimentar) con el tipo de colores que se ocupen. Los colores que se utilizaron fueron los normales, de madera, crayola y colores fluorescentes. Asimismo, se utilizaron objetos autoadheribles fosforescentes. Estos últimos colores que menciono son muy utilizados en la escuela, en particular en la primaria: son de uso familiar los marcatextos (fluorescentes) y cartulinas con colores muy brillantes (fluorescentes, también). Los objetos autoadheribles son estampas que brillan en la oscuridad y que pueden pegar en sus habitaciones. Esto es fundamental para la actividad, dado que estamos utilizando materiales que son conocidos por los alumnos y que se observan en un ambiente diferente y preparado.

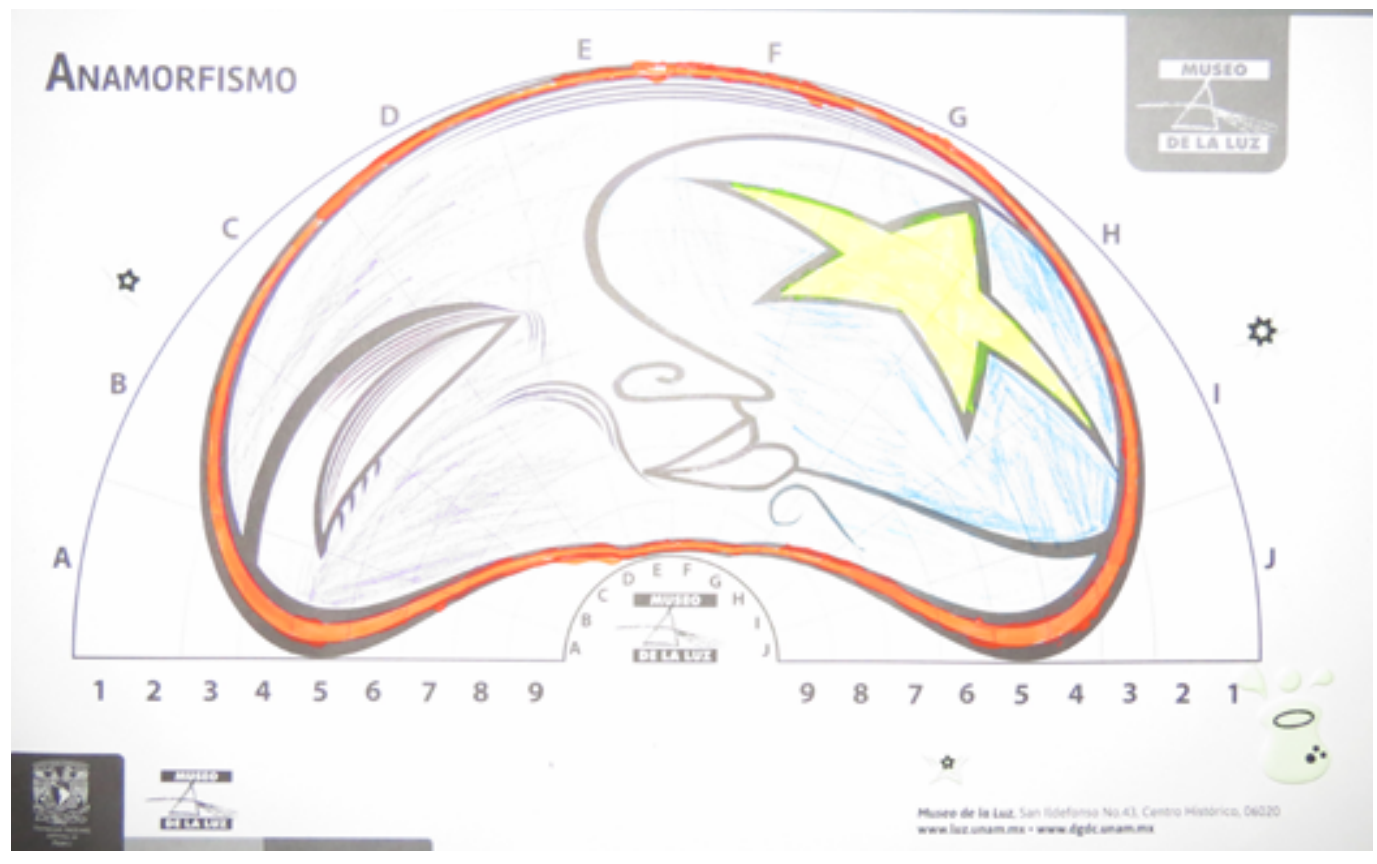



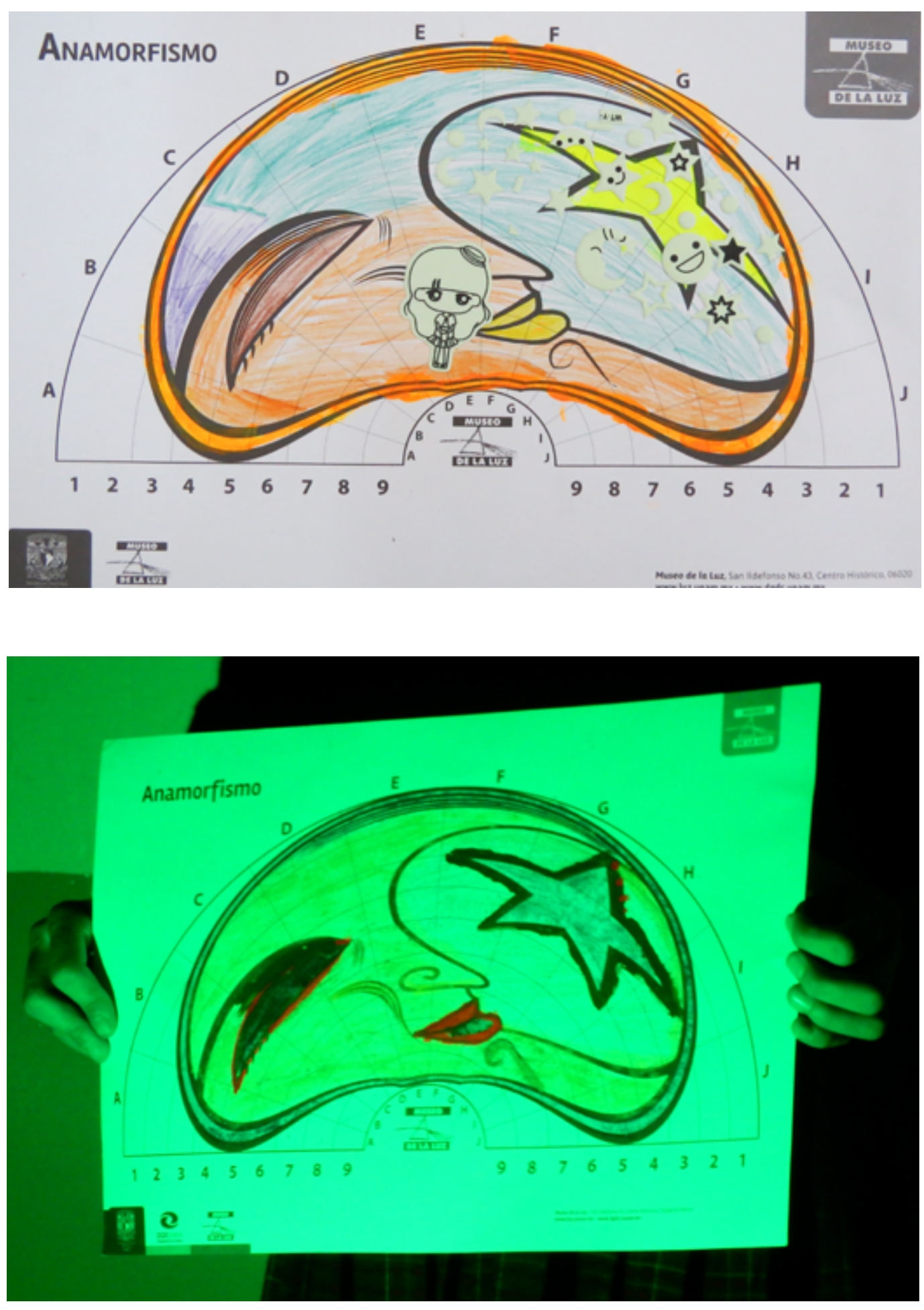

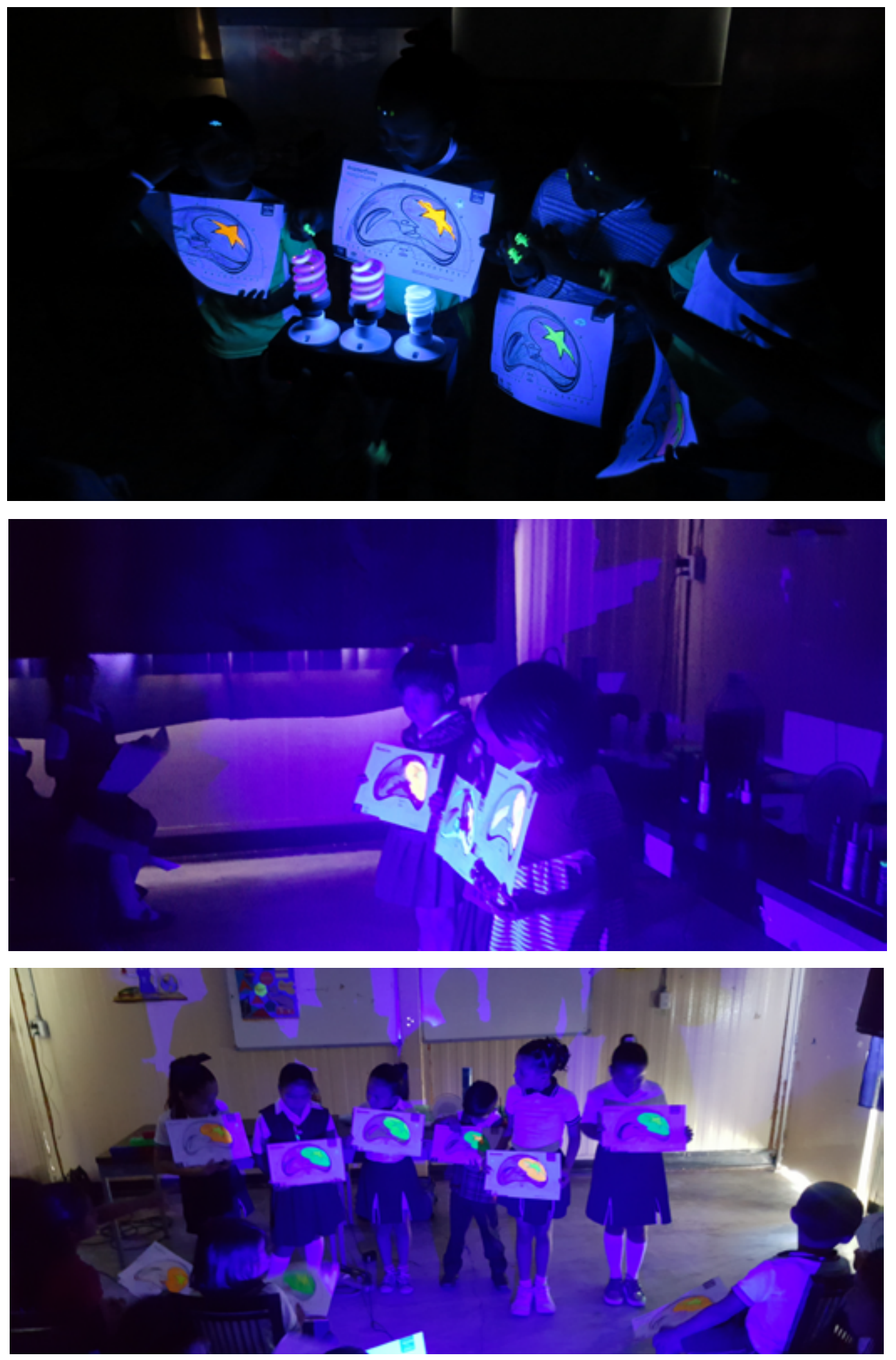

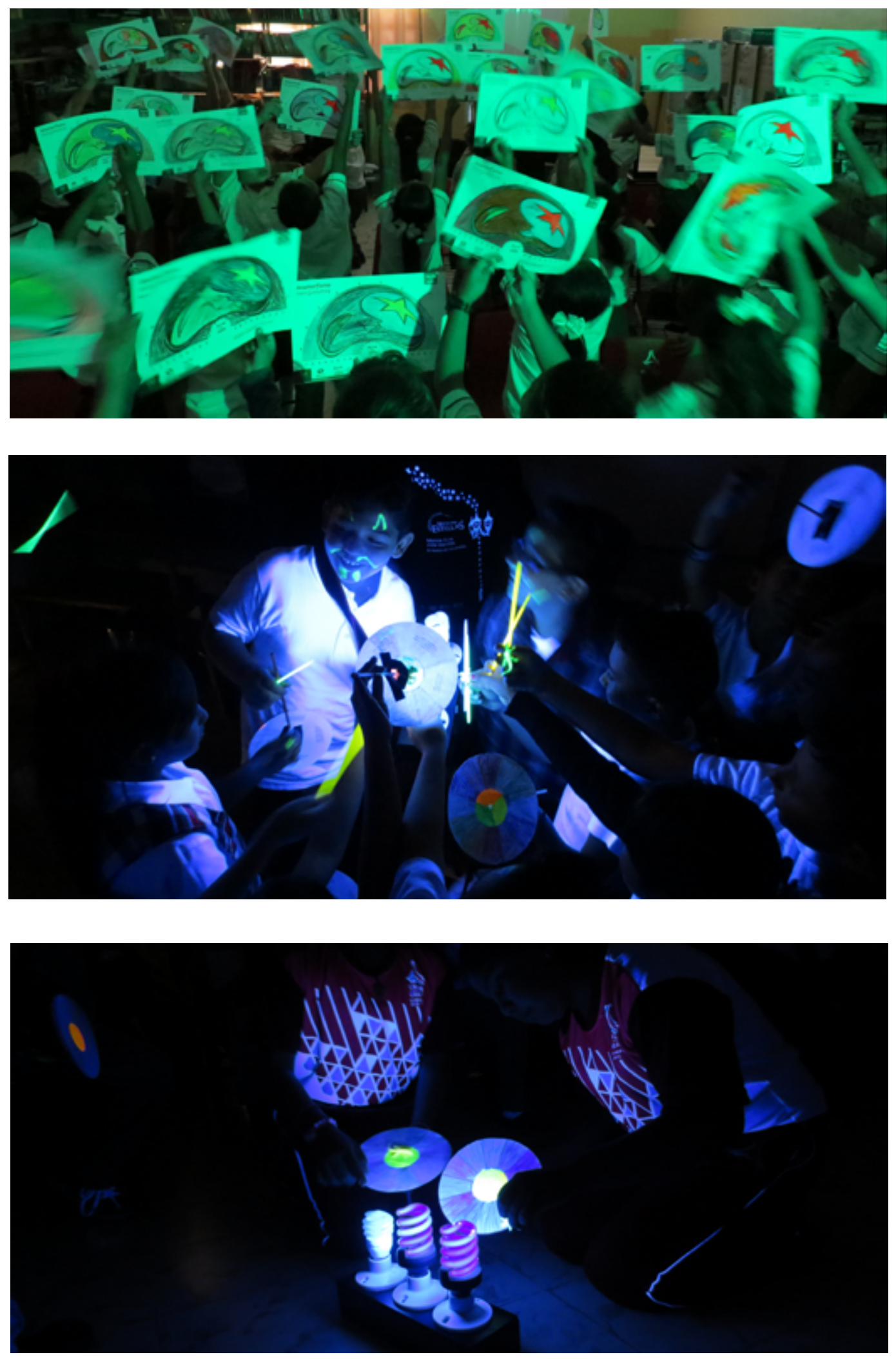


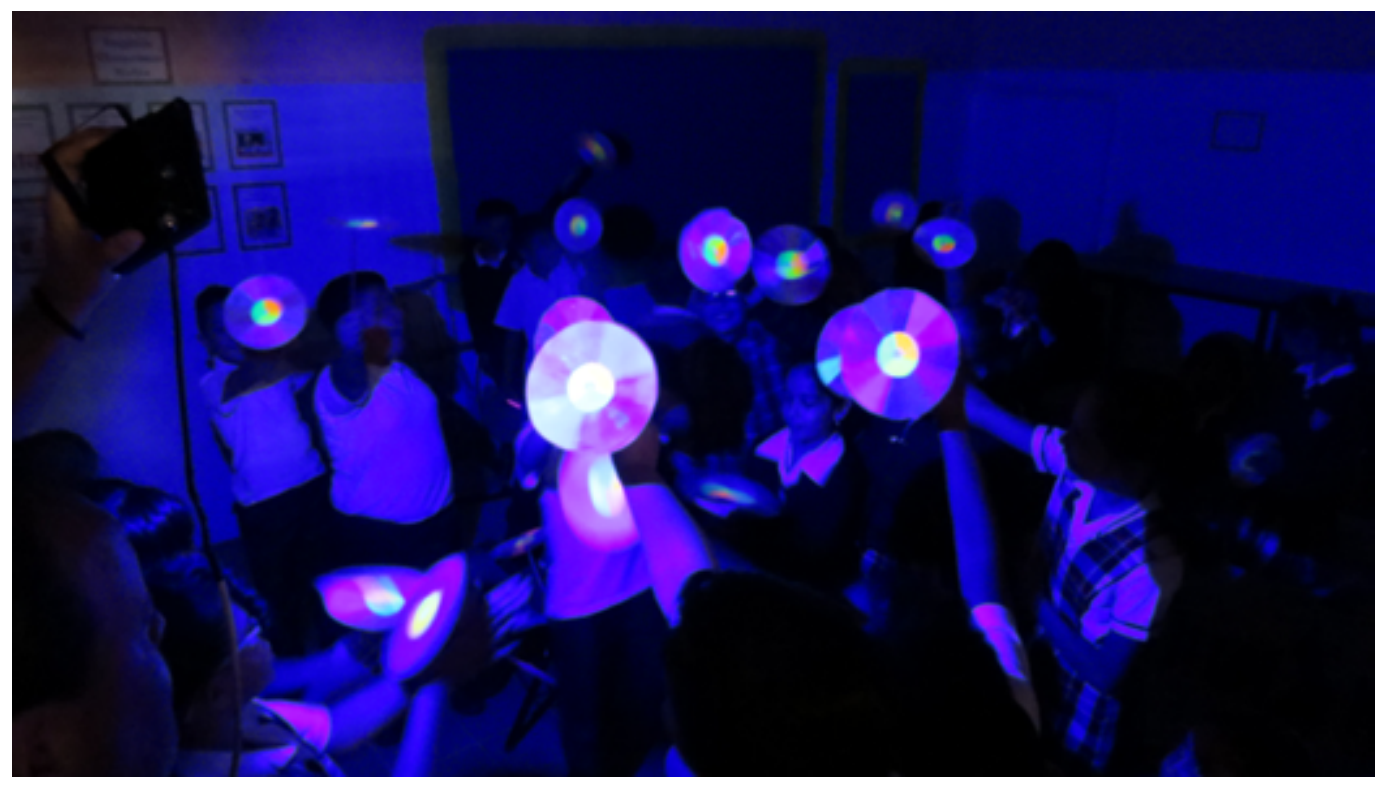

El ambiente especial anteriormente mencionado lo creamos en la demostración de luminiscencias, la otra actividad de ciencia que se desarrollaba en las escuelas. Se oscurecía un salón y colocábamos lámparas especiales (lámparas de luz negra o uv), focos normales (la bombilla incandescente y la lámpara fluorescente compacta), además, una lámpara de leds la cual podíamos cambiar el color de la luz a nuestra conveniencia. Como parte de la demostración, dichas fuentes de luz hacían que dos líquidos al juntarlos emitieran luz (quimiluminiscencia). Es decir, en el salón oscuro los alumnos veían y experimentaban que existen varios mecanismos de generar luz, no nada más las conocidas y las más normales.

La primera actividad de ciencia que se realizaba era el taller. Pintaban el Disco de Newton o el Anamorfismo utilizando los distintos colores, dependiendo del grado escolar: para los alumnos de $1^{\circ}, 2^{\circ}$ y $3^{\circ}$ el Anamorfismo, y $4^{\circ}, 5^{\circ}$ y $6^{\circ}$ el Disco de Newton. Pero antes de comenzar, jugaban con prismas y espejos de tal suerte que tenían que meter un haz de luz y, con el prisma, generar el espectro visible. Ese momento de experiencia y observación fue uno de sus primeros asombros: el ver cómo se produce los colores a partir de la refracción de la luz por un prisma.

En el caso de que no fuera posible la anterior actividad, con el prisma se les pedía que vieran hacia una fuente luminosa, un foco o una lámpara del salón. Esa experiencia nos servía para comenzar el diálogo, el juego y motivar la curiosidad innata de los niños. Se hacía énfasis en que el color que percibimos de todo lo que nos rodea es gracias a que en la luz vienen todos los colores, lo que observamos es el resultado de esa luz, ya sea de un foco o la del Sol, interactuando con el material y objeto que observamos. Les hacíamos preguntas como: ¿qué color observarían de algo si la luz que incide en él estuviera compuesta de un solo color?, ¿el color que percibimos de un objeto es una característica exclusiva de él?, ¿qué papel juega la luz en todo esto? Para pintar su Disco de Newton 
o Anamorfismo les proporcionábamos colores fluorescentes, además de los normales. En ese momento no les decíamos las características y propiedades de una sustancia o pintura fluorescente, simplemente la experiencia era que observaran cómo unos colores brillan más que otros. En todas las sedes a las que fuimos, lo interesante fue que los alumnos conocían estos materiales, estas pinturas, estos marcatextos pero siempre había la confusión entre algo fluorescente y fosforescente, la evidencia era que unos colores brillan más que otros y hay objetos que brillan en la oscuridad. Los objetos que los alumnos elaboraron posteriormente los iban a observar en la demostración de luminiscencias, en el salón previamente oscurecido y con lámparas especiales. El asombro fue total: el jwowww! resonaba al unísono en el salón.

Lo primero que se hacía en el salón de la demostración de luminiscencia era mostrar, con diferentes focos o lámparas, que no todos los focos se calientan al estar emitiendo luz. Aunque parece una afirmación evidente, no lo es del todo cuando tocas o intentas tocar los focos encendidos. Todos conocen, por experiencia, que los focos de bombilla incandescentes cuando están emitiendo luz no se pueden tocar dado que están calientes. Pero no es tan conocido que las lámparas fluorescentes compactas (LFC) no se calientan. Al niño lo hacíamos tocar la lámpara y en ese momento la prendíamos, el niño sentía y veía que al estar emitiendo luz no se calentaba lo suficiente como para retirar las manos inmediatamente. Con los focos incandescentes bastaba con prenderlo unos segundos, después apagarlo, y decirle al niño que acercara la mano. Nunca llegaron a tocarlo porque sentían la emisión de energía térmica.

Dado que los alumnos llegaban con lo que habían elaborado en su taller, lo siguiente que se hacía era que observaran lo que pintaron, pero con un foco especial, una lámpara de luz negra. Como el salón estaba oscurecido, apagábamos todas las luces y veían que lo que habían hecho en el taller requería de luz para verlo, de hecho, se requiere de luz para poder ver todo lo que nos rodea. Al prender la lámpara de luz negra, observaban cómo la zona que habían pintado con la pintura fluorescente brillaba más que lo demás. Recordemos que en taller se usaron estampas fosforescentes, de tal suerte que cuando se apagaba la luz negra la pintura fluorescente dejaba de brillar y continuaba brillando la estampa fosforescente. Por lo tanto, los alumnos experimentaban en carne propia, y menciono carne propia porque literalmente eso se hacía, se les pintaba sus manitas o su carita con el marcatextos (plumón fluorescente) y se les colocaba una estampa fosforescente. Entonces observaban qué sucedía con los materiales fluorescentes y fosforescentes cuando la lámpara de luz negra estaba encendida, y lo que pasaba cuando la lámpara se apagaba. Esa era una gran experiencia y era el momento de comentarles la diferencia ente un fenómeno fosforescente y uno fluorescente (lo fosforescente brilla en la oscuridad y lo fluorescente brilla sólo mientras la luz negra esté encendida); además, tenían el ejemplo de algo que brilla, o está emitiendo luz, y no está caliente. Esto era sólo el principio en la demostración. 
A continuación, experimentaban con dos sustancias líquidas: agua oxigenada y luminol. Pasaban al frente y cuando vertían un líquido en el otro en oscuridad, la reacción química que se genera produce luz. Los alumnos quedaban totalmente sorprendidos. Posteriormente, les dábamos las varitas o pulseras quimiluminiscentes que, en lo general, ya conocían, ocasionalmente en las fiestas dan estas varitas como parte de la diversión o inclusive, algunos alumnos, mencionaban que las habían visto y jugado con ellas en paletas de dulce. Antes de concluir, se hablaba de organismos vivos que tienen la propiedad de emitir luz y todos participaban contestando el nombre de esos bichos: luciérnagas, cocuyos, ciertos peces marinos, medusas y hasta de los hongos se habló.
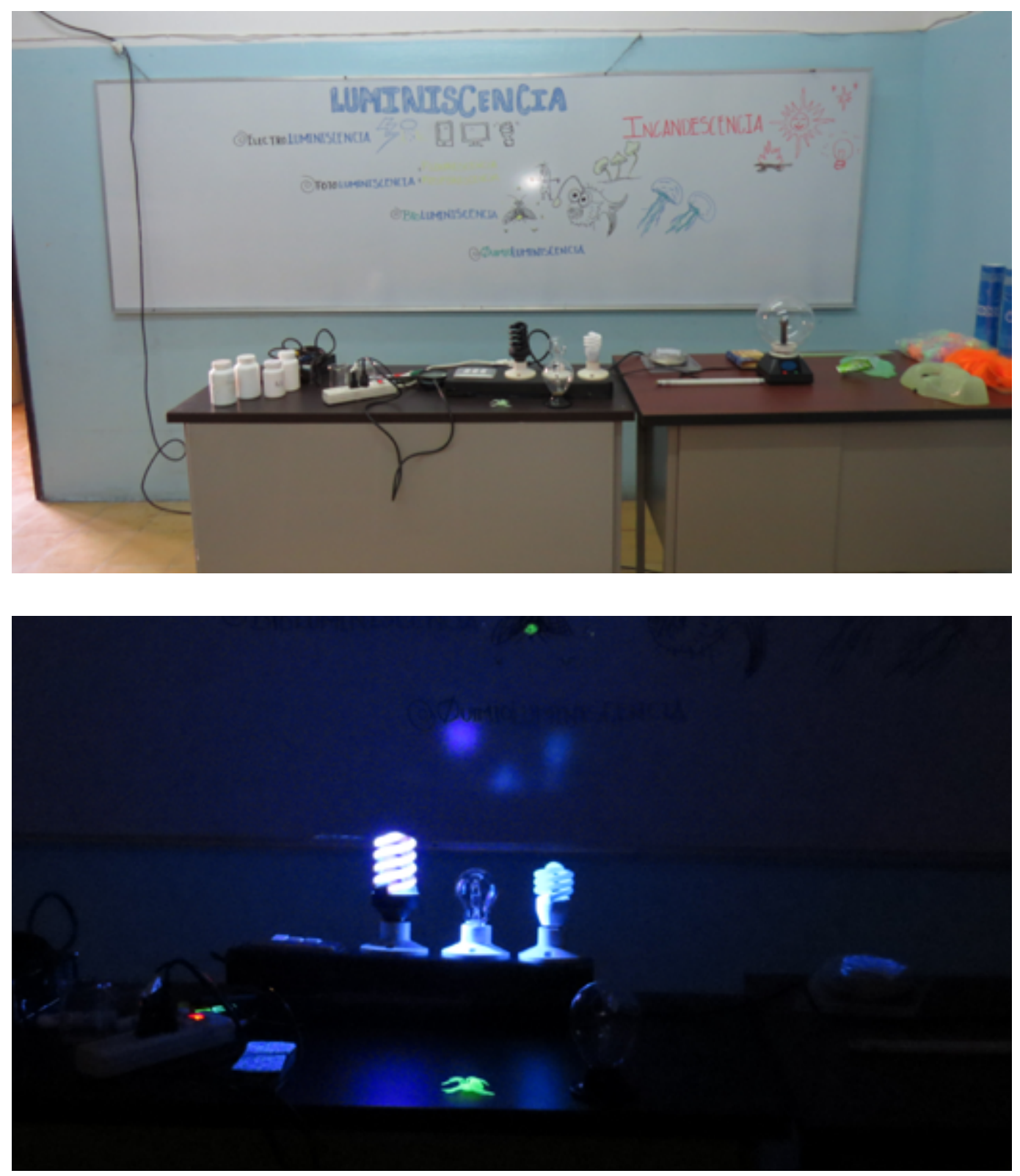

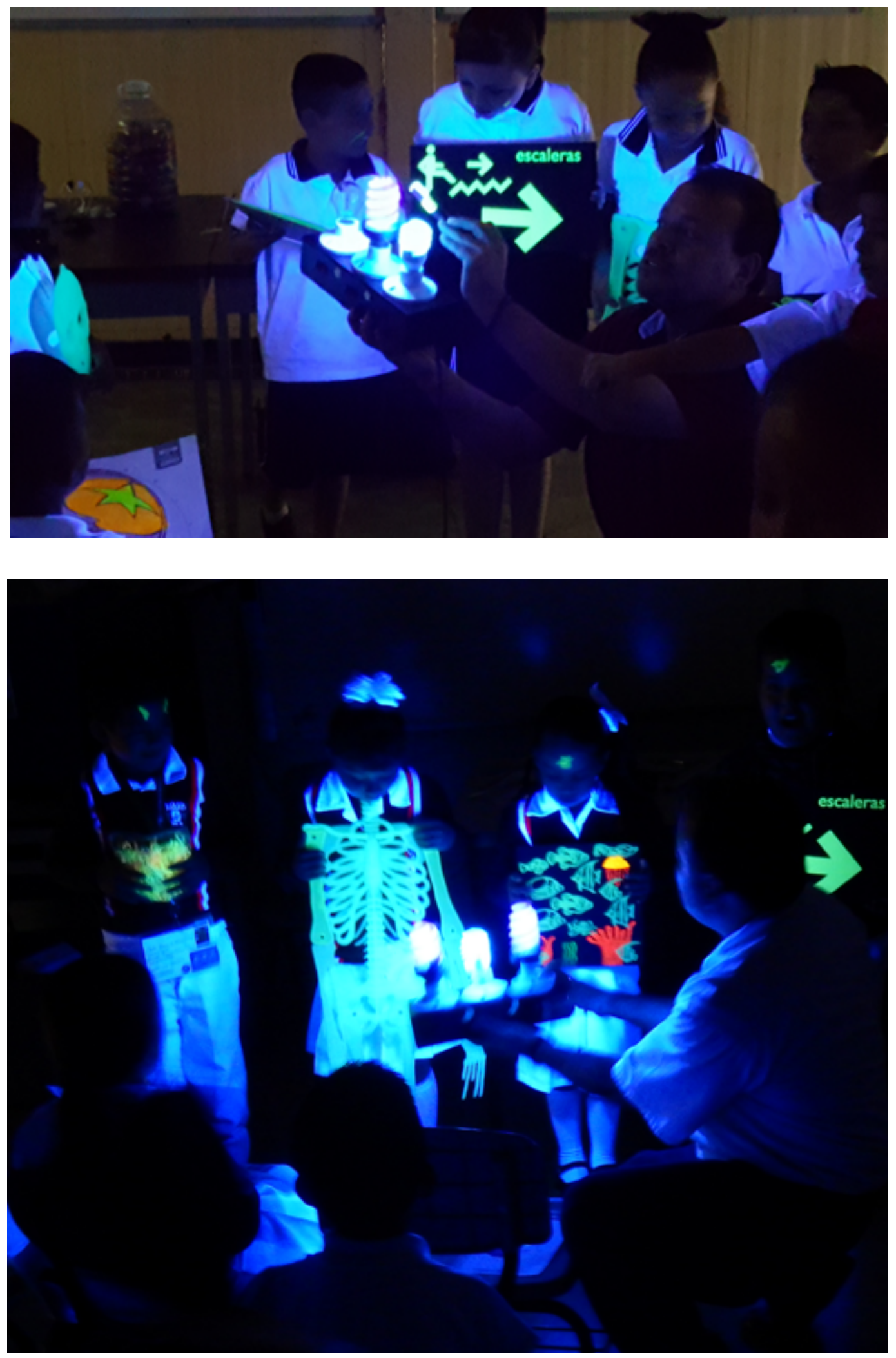

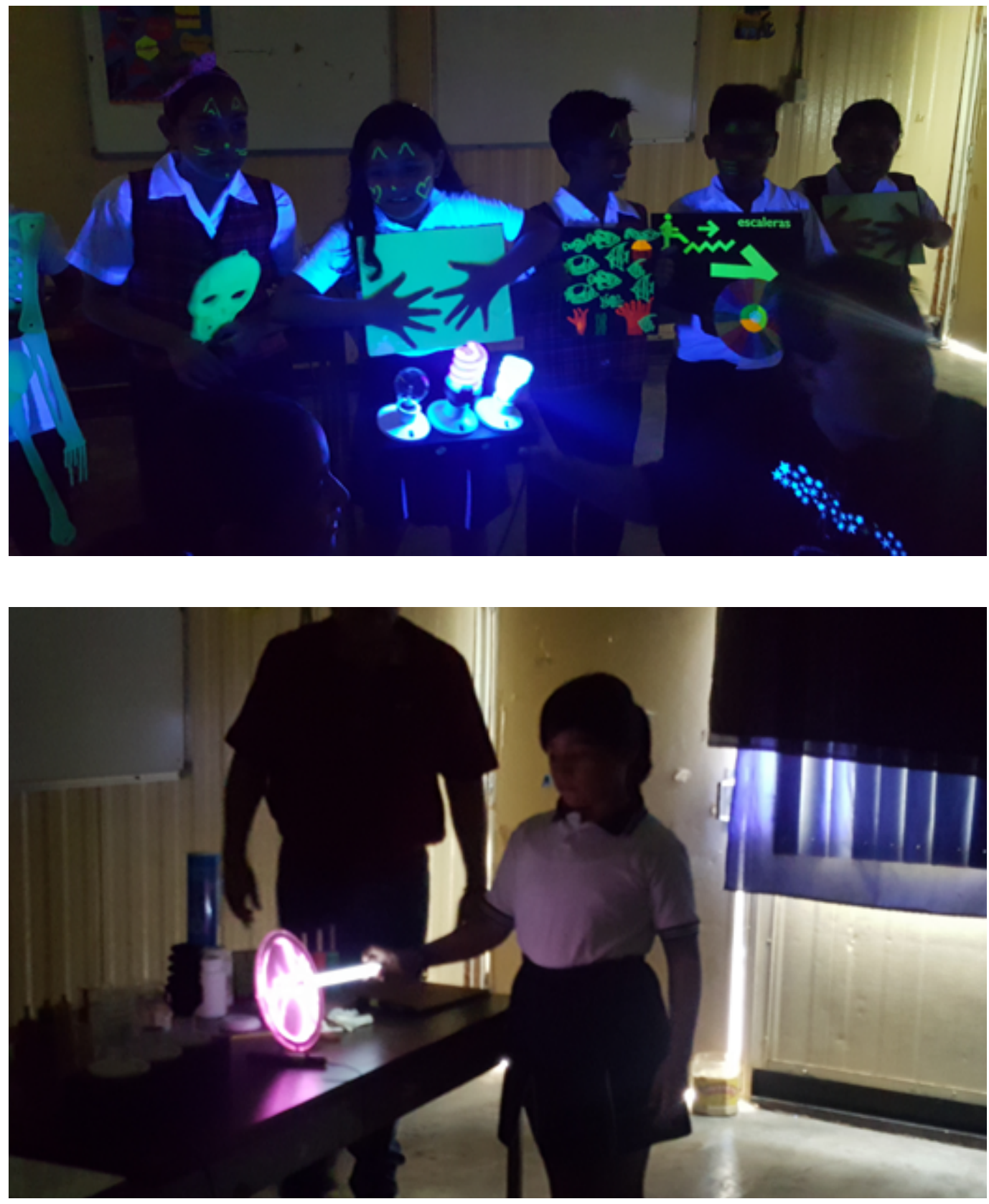

Para finalizar, observaban lo que habían elaborado con una lámpara de led en la que se puede controlar el color de luz que emite. Tres colores son los importantes que emiten estas lámparas y que nosotros utilizamos: rojo, verde y azul. Cuando observaban su trabajo con pura luz roja, el aspecto del color cambiaba a cuando se observaba con luz verde y, no se diga, cuando se iluminaba con la azul. Con esta experiencia quedaba clara la importancia de la luz en la percepción del color de los objetos, cómo el color no es propiedad exclusiva del objeto, se hacía evidente el papel fundamental de la luz y de su naturaleza. 
En las seis sedes que visitamos con esta actividad, se vio la misma admiración en los niños. De primero a sexto grado los comentarios fueron de sorpresa. En los salones que utilizamos para la demostración siempre había materiales de todo tipo de pintura, una gran variedad de color y objetos fluorescentes o fosforescentes o ambos, cartulinas, hojas, aros de colores, hasta un frisbee fosforescente. Por tanto, los alumnos podían ahora clasificar todo lo que observaban y usar correctamente ciertos términos científicos. Cuando los alumnos salían de la escuela y llevaban lo que hicieron en su taller y los objetos fosforescentes, fluorescentes, quimiluminiscentes, inmediatamente comentaban con sus padres lo que habían hecho.

Los artistas plásticos y una selección reducida de alumnos crearon un mural con una selección reducida de alumnos, en el que utilizaron pinturas regulares y las brillantes. Se colocaron lámparas de luz negra para que se observara el mural y los efectos lumínicos producidos por las lámparas. Los alumnos, seguramente, cuando veían la obra, recordarían lo vivido.

Para el Museo de la Luz, este binomio, divulgadores y artistas, no se había presentado. No se había tenido la oportunidad de juntos participar en un proyecto. El Museo de la Luz ha participado desde su inicio, hace veintiún años, en diversas actividades fuera del museo. Se llevan actividades a diferentes escuelas de educación básica, media superior y superior; así como, a diferentes eventos culturales que se organizan en la Ciudad de México y en el interior de la república mexicana. Sin embargo, la experiencia de estas seis escuelas ha sido muy enriquecedora y se han aprendido muchas cosas nuevas. Las actividades extramuros regulares tienen como fin mostrar el fenómeno de la luz con talleres y demostraciones diversas, el eje fundamental es la luz. En esta ocasión, no sólo fue así, sino que se buscó dejar en los niños la inquietud por la experimentación, la indagación y aprovechar su curiosidad natural. Nos centramos en el color, en la forma en que se genera, la importancia de la luz para ello; también, en mostrar los mecanismos diferentes que hay para producir luz. El hecho de que después los niños hayan desarrollado un mural y que en él se exponen las experiencias que vivieron en las actividades, además de referentes regionales y culturales, ha hecho que este proyecto tenga un toque especial.

\section{Conclusión}

En mi opinión, y en estos más de 26 años que llevo desarrollando actividades similares, el haber participado en este proyecto me ha abierto una posibilidad que no había explorado: hablar de la ciencia con el pretexto del desarrollo de una pieza artística. Normalmente, a los diversos lugares que he ido, siempre era la luz, y el conocimiento común que se tiene de ella, quien llevaba la pauta en el desarrollo de la charla, la conferencia, el taller, la demostración o cual fuere la estrategia o el proceso para que, al final de la actividad, se viera a la luz desde 
otra perspectiva, que se viera a la luz, además de familiar, con una mirada de curiosidad y generadora de una gran cantidad de fenómenos que vemos, admiramos y utilizamos a diario.

Cabe mencionar que, para diseñar o planear la estrategia en esta ocasión, comencé exponiendo, de manera informal, y como siempre lo hago, el tema de la luz a los artistas plásticos que participarían en el proyecto. Para ellos también era una experiencia nueva. Para determinar su pieza y la forma de trabajo que iban a desempeñar en las escuelas, tomaron ideas de lo que experimentaron en esa plática informal. Yo, por mi parte, al saber que el trabajo iba a ser conjunto, tenía que lograr que las niñas y los niños, cuando vieran lo creado, principalmente tuvieran una experiencia de gozo y admiración con la actividad de ciencia, como se tiene, normalmente, cuando se observa una pieza de arte. Ese fue el reto principal en el que me enfoqué. En el proceso de las actividades siempre tratamos de crear admiración y contemplación por los efectos ópticos observados y tener presente que eso que observaban y experimentaban se plasmaría de manera natural en una pieza artística. Me siento totalmente complacido de haber vivido esta experiencia y ahora poder compartirla; la ciencia y el arte, el arte y la ciencia siempre van de la mano.

\section{Cómo citar este artículo}

* Hernández Valencia, Isaías (2018). A todas luces, una experiencia única entre ciencia y arte. Revista Digital Universitaria (RDU). Vol. 19, núm. 3 mayo-junio. DOI: http://doi.org/10.22201/codeic.16076079e.2018.v19n3.a6. 